\title{
Correction of Misclassifications Using a Proximity-Based Estimation Method
}

\author{
Antti Niemistö \\ Institute of Signal Processing, Tampere University of Technology, P.O. Box 553, 33101 Tampere, Finland \\ Email: antti.niemisto@tut.fi \\ Department of Pathology, The University of Texas M.D. Anderson Cancer Center, 1515 Holcombe Boulevard, \\ Houston, TX 77030, USA

\section{llya Shmulevich} \\ Department of Pathology, The University of Texas M.D. Anderson Cancer Center, 1515 Holcombe Boulevard, \\ Houston, TX 77030, USA \\ Email: is@ieee.org
}

\section{Vladimir V. Lukin}

Department 504, National Aerospace University, 17 Chkalova Street, 61070 Kharkov, Ukraine Email:lukin@ai.kharkov.com

\author{
Alexander N. Dolia \\ Department 504, National Aerospace University, 17 Chkalova Street, 61070 Kharkov, Ukraine \\ School of Electronics and Computer Science, University of Southampton, Southampton SO17 1BJ, England, UK \\ Email:ad@ecs.soton.ac.uk

\section{Olli Yli-Harja} \\ Institute of Signal Processing, Tampere University of Technology, P.O. Box 553, 33101 Tampere, Finland \\ Email: olli.yli-harja@tut.fi
}

\section{Received 14 October 2003; Revised 17 December 2003; Recommended for Publication by John Sorensen}

\begin{abstract}
An estimation method for correcting misclassifications in signal and image processing is presented. The method is based on the use of context-based (temporal or spatial) information in a sliding-window fashion. The classes can be purely nominal, that is, an ordering of the classes is not required. The method employs nonlinear operations based on class proximities defined by a proximity matrix. Two case studies are presented. In the first, the proposed method is applied to one-dimensional signals for processing data that are obtained by a musical key-finding algorithm. In the second, the estimation method is applied to two-dimensional signals for correction of misclassifications in images. In the first case study, the proximity matrix employed by the estimation method follows directly from music perception studies, whereas in the second case study, the optimal proximity matrix is obtained with genetic algorithms as the learning rule in a training-based optimization framework. Simulation results are presented in both case studies and the degree of improvement in classification accuracy that is obtained by the proposed method is assessed statistically using Kappa analysis.
\end{abstract}

Keywords and phrases: misclassification correction, image recognition, training-based optimization, genetic algorithms, musical key finding, remote sensing.

\section{INTRODUCTION}

Automatic classification of data is a standard problem in signal and image processing. In this context, the overall objective of classification is to categorize all data samples into different classes as accurately as possible. The selection of classes depends naturally on the particular application. Powerful supervised classification methods based on neural networks, genetic algorithms, Bayesian methods, and Markov random fields have been developed (see, e.g., $[1,2,3]$ ). However, even the most advanced methods of automatic classification are typically unable to provide a classification without 
misclassifications. The main reason for this is the inherent presence of noise in data as well as the structure of the signals and images themselves. Usually, the larger the noise level (variance), the greater the probability of misclassifications.

This problem, however, is almost always approached from the point of view of feature selection and classifier design. For example, the emphasis is usually placed on the synthesis of powerful one-stage classification methods. Unfortunately, in many cases, the original data or the classifier itself may not be made available and hence cannot be improved upon. Thus, there is a need to be able to correct misclassifications.

We propose a nonlinear estimation method for the misclassification correction task. Some preliminary results have been published earlier in our conference papers $[4,5,6,7,8]$. The idea is that the classification procedure consists of two separate stages: the primary classification (recognition) stage and the misclassification correction stage. The basic philosophy behind the proposed method is that contextual information should be utilized, implying that neighboring samples can carry useful information about each other. This is the conventional view in most signal/image processing applications, especially in filtering, where information in a sliding window is used to estimate the central pixel. Markov random field modeling approaches to image processing are also based on utilization of spatial dependencies and have been successfully used for classification of images [3].

The fundamental difference between the proposed estimation method and traditional signal/image filtering methods is that the latter are essentially quantitative in that they operate on sample values or other quantitative information, whereas the proposed method is applied to categorical data in which every sample may belong to one of a number of possible classes. As such, categorical data contain structural information, but can be thought of as taking values drawn from some finite alphabet representing class membership. One obvious approach might be to map these symbols to integers and then subsequently employ traditional signal/image processing methods designed for numerical data. The difficulty with such an approach is that the choice of the mapping can have a drastic effect on the subsequent analysis. Another approach is to remain entirely in the symbolic or categorical domain. There have been very few totally symbolic signal processing approaches, with the notable exception of [9], where the authors show how to compute spectrograms with an application to DNA sequence analysis. The proposed method also operates entirely in the categorical domain.

The proposed method makes use of the notion of class proximities. Informally speaking, two classes are close to each other if the samples belonging to these classes are likely to be found in temporal proximity to each other in a onedimensional (1D) signal or in spatial proximity to each other in a two-dimensional (2D) image. In this way knowledge of the classes themselves can be used, which is a fundamental advantage over simpler approaches, such as weighted majority filters. The mathematical formulation of the proposed method is closely related to the so-called median function on graphs [10], which plays a role in consensus theory [11].
The proposed estimation method is discussed in detail in Section 2. This is followed by two case studies. The case study of section 3 illustrates the use of the proposed method in $1 \mathrm{D}$ signal processing and takes its example from musical key-finding, whereas the case study of section 4 applies the method in 2D to correct misclassifications in classification of remotely sensed images. The use of training-based optimization for finding the optimal proximity matrix employed by the method is also discussed, and Kappa analysis is employed to statistically assess the degree of improvement in classification accuracy that is obtained by the proposed method. Finally, some concluding remarks are given in Section 5.

\section{ESTIMATION METHOD}

It is well known that in the case of real numbers, the $L_{p}$-norm estimate of $X=\left\{x_{1}, x_{2}, \ldots, x_{N}\right\}, x_{i} \in \mathbb{R}$ is the value $\beta$ that minimizes [12]

$$
\sum_{i=1}^{N}\left|x_{i}-\beta\right|^{p}
$$

For example, the median operator can be defined as

$$
\operatorname{med}(X)=\arg \min _{\beta \in X} \sum_{i=1}^{N}\left|x_{i}-\beta\right|
$$

when $N$ is odd. Similarly, the mean operator can be defined as

$$
\operatorname{mean}(X)=\arg \min _{\beta \in \mathbb{R}} \sum_{i=1}^{N}\left(x_{i}-\beta\right)^{2} .
$$

Suppose that instead of a set of real numbers we have some multiset of samples of class data $B=\left\{b_{1}, b_{2}, \ldots, b_{N}\right\}$. Then, analogous to the $L_{p}$-norm, we can define

$$
C_{p}(B)=\arg \min _{\beta \in B} \sum_{i=1}^{N} w\left(\beta, b_{i}\right)^{p},
$$

where $w$ is a proximity function. The value $p=1$ corresponds to the "median" and $p=2$ to the "mean" of the samples in $B$. Note that the estimate (4) is necessarily one of the samples in $B$.

The proximity function $w$ can be arbitrary, which means that the requirements of a metric are lifted. This is exactly why we talk about interclass proximities and not distances. Suppose the set of possible classes is $V=\left\{v_{1}, v_{2}, \ldots, v_{n}\right\}$. For example, $w\left(v_{i}, v_{j}\right)$ is the proximity from $v_{i}$ to $v_{j}$. The greater the proximity from one class to another, say from $v_{i}$ to $v_{j}$, the less likely it is that $v_{i}$ occurs in the neighborhood of $v_{j}$. Note in particular that the proximities do not have to obey the triangle equality. This is important, because it is clear that it can be very likely for $v_{i}$ to occur in the neighborhood of two different classes $v_{j}$ and $v_{k}$, while it may not be likely for $v_{j}$ to occur in the neighborhood of $v_{k}$. 
The proximity function can be represented by a proximity matrix $\mathbf{W}$ with each entry $w_{i j}$ equal to $w\left(v_{i}, v_{j}\right)$. Then, the power $p$ can be incorporated into the proximity matrix by replacing each entry $w_{i j}$ by $w_{i j}^{p}$. This makes (4) similar to a result stemming from the theory of median graphs [10]. In that context, a profile of length $N$ on a weighted graph $G(V, E)$ (where $V$ is the vertex set and $E$ is the edge set) is a finite sequence $\pi=\left\{v_{1}^{\prime}, v_{2}^{\prime}, \ldots, v_{N}^{\prime}\right\}$ of vertices of $G(V, E)$, and the median of $\pi$ is defined as

$$
\operatorname{med}(\pi)=\arg \min _{\beta \in \pi} \sum_{i=1}^{N} d\left(\beta, v_{i}^{\prime}\right),
$$

where $d$ is the usual shortest path distance between two vertices. The vertex set $V$ corresponds to the set of possible classes. If $G(V, E)$ is a complete graph with nonnegative weights, this definition is identical to (4) with the power $p$ incorporated into the proximity function.

The following example demonstrates the calculation of $C_{p}$. The example also illustrates how samples may be repeated in $B$, and thus $B$ is a multiset.

Example 1. Consider the proximity matrix

$$
\mathbf{W}=\left[\begin{array}{lll}
1 & 2 & 3 \\
3 & 1 & 4 \\
2 & 4 & 2
\end{array}\right]
$$

where the first row refers to the class $v_{1}$, the second to $v_{2}$, and the third to $v_{3}$. Suppose that we have the multiset of samples

$$
B=\left\{b_{1}, b_{2}, b_{3}, b_{4}, b_{5}\right\}=\left\{v_{1}, v_{1}, v_{2}, v_{2}, v_{3}\right\} .
$$

We compute $C_{1}(B)$. The proximity from $v_{1}$ to itself is 1 , the proximity from $v_{1}$ to $v_{2}$ is 2 and to $v_{3}$ is 3 . Thus, the sum of proximities from $v_{1}$ to all the samples in $B$ is $1+1+2+2+3=$ 9. Similarly, the sum of proximities from $v_{2}$ to all the samples in $B$ is $3+3+1+1+4=12$, and from $v_{3}$ the sum of proximities is $2+2+4+4+2=14$. Therefore, since the sum of proximities is minimum from $v_{1}, C_{1}(B)=v_{1}$.

Similarly to the running median and mean filters based on (2) and (3), we can define a sliding-window processing operation based on (4). This can be done by simply choosing $B$ to be the contents of the sliding window at each position. Then, we only need to calculate $C_{p}(B)$ and consider the result as the output of the operator at that window position. In $1 \mathrm{D}$, the processing operator can be expressed as

$$
y(i)=C_{p}[x(i-k), \ldots, x(i), \ldots, x(i+k)],
$$

where $\{x(i)\}$ is the sequence of input data, $\{y(i)\}$ is the sequence of output data, and the width of the sliding window is $2 k+1$. The generalization to $2 \mathrm{D}$ is straightforward. This definition leads to a nonlinear estimation method for class data. Analogously to median and mean filters, the obtained operator is a smoothing operator. Therefore, it is only applicable in situations where some continuity in the class data is expected.
It is also possible to weight the samples in the sliding window differently. This enables us to treat each sample differently depending on its location. The weighted modification of (4) can be written as

$$
C_{p}(B)=\arg \min _{\beta \in B} \sum_{i=1}^{N} a_{i} w\left(\beta, b_{i}\right)^{p},
$$

where $a_{i}$ is the weight of $b_{i}$. This definition is analogous to the definition of the weighted median filter [12]. If the spatial weights are natural numbers, weighting can also be thought of as duplication of samples in the same way as in weighted median filtering.

In some classification tasks the set of classes can be divided into two distinct subsets: the basic classes and the supplementary classes. The actual division of classes into basic and supplementary ones is dependent on the application. We call supplementary such classes that are needed only for providing additional information for the misclassification correction stage. The basic classes are then the proper classes, and they appear only in the final classification result that is obtained after correction of misclassifications. A simple modification to (9) provides this property. Let $B_{b}$ be the multiset of samples in the window that represent the basic classes and let $B_{s}$ be the multiset of samples in the window that represent the supplementary classes. Then $B=B_{b} \cup B_{s}$ and $B_{b} \cap B_{s}=\varnothing$, and we can define

$$
C_{p}\left(B, B_{b}\right)=\arg \min _{\beta \in B_{b}} \sum_{i=1}^{N} a_{i} w\left(\beta, b_{i}\right)^{p} .
$$

One alternative approach to postclassification smoothing is to use weighted majority filters [13]. In fact, the proposed method can be thought of as a generalization of this filter class. If the proximity matrix is a matrix of ones with a main diagonal of zeros, the output is the same as the output of the basic (unweighted) majority filter. However, determining the corresponding proximity matrix for a weighted majority filter may not be straightforward, and not all proximity matrices have a corresponding weighted majority filter. Moreover, it is in general not possible to select the weights of a weighted majority filter such that the filter preserves fine details and removes misclassifications at the same time. The following case studies illustrate how this can be achieved using the proposed method.

\section{CASE STUDY 1: CORRECTION OF MISCLASSIFICATIONS IN MUSICAL KEY FINDING}

In this case study, the proposed estimation method is applied in 1D for processing data that are obtained by a musical key-finding algorithm. The musical key is a system of pitches and harmonics generated from a scale of tones, one of which is predominantly important. In total, there are 24 keys, 12 major and 12 minor. Although a key is typically indicated at the beginning of a piece of music by means of a key signature, which sets up the overall tonal organization and 
center, compositions often move away from the fundamental key and shift into other keys. Here, our goal is to detect these shifts and to establish the key in a local region in a piece of music.

The key-finding algorithm and the obtained data are discussed in Section 3.1. The algorithm can be applied to a musical composition to find a sequence of local key assignments. However, such a sequence typically contains many oscillations and impulses. As is discussed in Section 3.2, the keys can be thought of as classes, and the proposed estimation method is directly applicable for smoothing the obtained signal.

\subsection{Musical key finding}

Automated key finding in music is a prerequisite to successful automation of music analysis. Specifically, the determination of key is necessary for meaningful coding of melodic and harmonic events [14]. A number of approaches have been shown to be successful for assigning a key signature to a musical composition $[14,15,16,17]$. A review of several other approaches can be found in [14]. Our objective, however, is not to find the key signature of a musical composition, but rather to trace the varying tonal orientations and modulations throughout the composition. Here, only information that is localized around the desired regions is used in determining the key. The motivation in this kind of local key finding is that information on the local key is needed in recognition of musical patterns $[18,19,20]$. Such a recognition system is useful in automatic retrieval of music information from large music databases.

Specifically, our goal is to be able to determine the primary key in a region centered around an arbitrary position in the score. An obvious approach is to determine the key of each region of a given length and then assign it to the note around which the region is centered. This does not mean that we are determining the key of a given note, which would be meaningless. Rather, we are merely using notes as location markers of regions. In this case study, the primary key is determined by using an algorithm proposed by Krumhansl [14] with the modification of using it in a sliding-window fashion instead of using it with nonoverlapping windows. The details of our approach can be found in [19].

The algorithm results in a sequence of class data. The classes correspond naturally to the 24 possible keys (12 major and 12 minor). The sequence of key assignments produced by sliding a key-finding window of length 7 notes over the right-hand part of Bach's Invention No. 8 in F-Major is shown in Figure 1. The keys are numbered arbitrarily from 1 to 24 , where the numbers 1 to 12 correspond to chromatic ordering of the major keys (C, C\#, D, etc.) and 13 through 24 correspond to minor keys. In a graphical depiction of a sequence of key assignments, such as Figure 1, a modulation appears as a step.

As can be seen, there is a lot of variation in certain regions of the sequence of key assignments. These artifacts often take the form of impulses or oscillations, especially in the regions of modulations. It must be stressed here, however, that the amplitudes of these impulses and oscillations do not

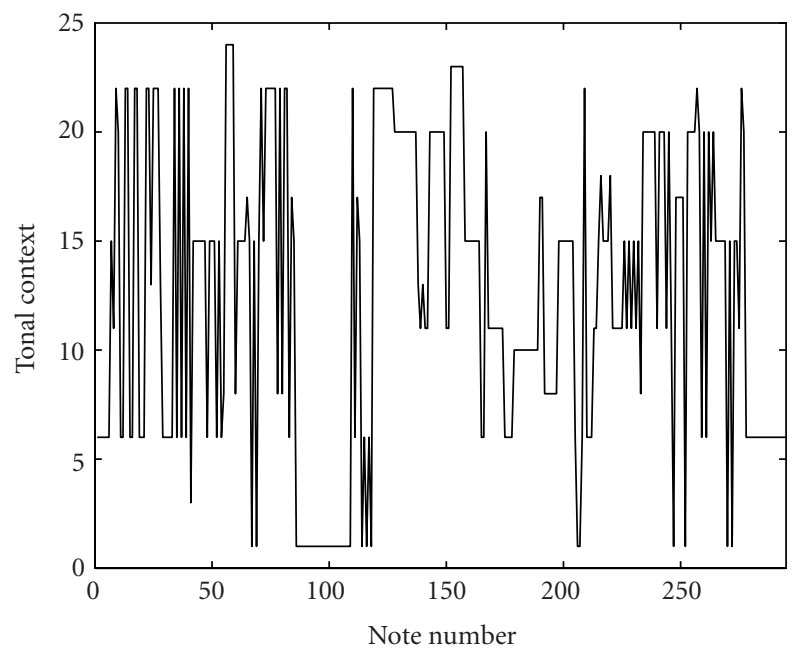

Figure 1: The sequence of key assignments for Bach's Invention No. 8 in F Major. The length of the key-finding window is 7 notes.

have a real meaning, because we are dealing with class data and the numerical representation of classes was chosen arbitrarily. Nonetheless, these artifacts certainly mean that the sequence exhibits undesired variability, which indicates that there are misclassifications. This is a fundamental property of the key-finding algorithm, especially if the key-finding window is small. In other words, the algorithm cannot be tuned to produce smooth sequences of key assignments without sacrificing specificity, and therefore we are forced to correct the misclassifications by some subsequent smoothing operation.

\subsection{Correction of misclassifications}

One approach for smoothing the obtained sequences of key assignments is to use the numerical representation of keys and to resort to the use of nonlinear filters such as the recursive median filter [21]. However, the obvious disadvantage in this approach is that the numerical representation was chosen arbitrarily and does not have a real meaning. In fact, there is no natural ordering of the keys since we are dealing with purely nominal class data. The result of smoothing by using a (nonlinear) filter thus depends on the artificial numbering of keys, and therefore a method based on filtering cannot be considered as a method providing correction of misclassifications.

A method that operates on class data is thus needed. Our approach here is to employ the spatially unweighted operator defined by (4). Naturally, each of the 24 keys is a class and the $24 \times 24$ proximity matrix represents so-called interkey distances. This approach is essentially similar to the approach based on median filtering: it is a local operation that takes into account the neighboring samples in choosing the output. The important distinction is that here we operate purely on class data and do not rely on any artificially imposed ordering of the keys. Moreover, as is explained below, the interkey distances can be chosen such that they represent the perceived proximity between keys. In that sense, our 
approach provides a more perceptually valid solution than the use of median-based filters.

In [22], correlations between the key profiles were used as a measure of interkey distance. A high correlation corresponds to a high degree of similarity between two keys while a low or negative correlation corresponds to a low degree of similarity. Then, the correlations can be used to produce a spatial representation of distances between keys by using multidimensional scaling [23]. Specifically, in this case multidimensional scaling results in a set of coordinates in a fourdimensional Euclidean space for each of the keys. Two keys that have a high degree of similarity correspond to points which are close to each other. More precisely, the Euclidean distances in the spatial configuration represent interkey distances.

The obtained interkey distances define the proximity matrix used by the estimation method. For example, the coordinate of $\mathrm{C}$ major is

$$
[0.567,-0.633,-0.208,0.480]
$$

and the coordinate of A minor is

$$
[0.206,-0.781,-0.580,0.119] \text {. }
$$

Then, the Euclidean distance between these two keys is 0.6488, which is equal to the corresponding element in the proximity matrix.

The following example illustrates the application of the proposed estimation method to correction of misclassifications in musical key finding.

Example 2. Suppose that the sliding window contains the following five key assignments: [C major, $\mathrm{C}$ major, $\mathrm{C \#}$ major, $\mathrm{C}$ major, A minor]. We estimate the key assignment using (4) with $p=1$. For each of the five keys, the sum of the distances to each key is calculated. In order, they are

$$
\text { [2.4491, 2.4491, 7.0923, 2.4491, 3.6377]. }
$$

Then, the output is the key corresponding to the vertex which has the minimum sum of distances. In this case the estimated key is $\mathrm{C}$ major.

To test the effectiveness of the proposed estimation method in correcting misclassifications in the case of sequences of key assignments, we apply it to Bach's Prelude in C Minor from Book II of the Well-Tempered Clavier, which was also studied by Krumhansl [14]. The sequence of keyassignments that is produced by the key-finding algorithm with a window length of 75 is shown in Figure 2. This window length is approximately equal to three measures, a choice that was also made by Krumhansl. The result of applying the proposed estimation method to the sequence of key assignments generated by the key-finding algorithm is shown in Figure 3. A window length of 99 was used. This choice enables us to remove any modulations that last less than 50 notes, or approximately two measures. Also, we choose $p=1$ in (4) in order to directly use the interkey distances and avoid

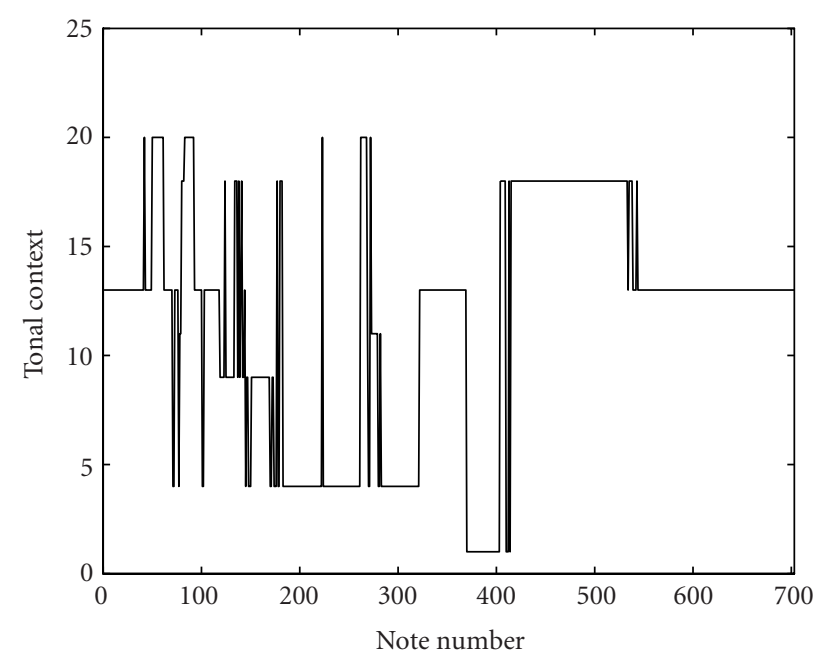

Figure 2: The sequence of key assignments for Bach's Prelude in C Minor from Book II of the Well-Tempered Clavier. The length of the key-finding window is 75 .

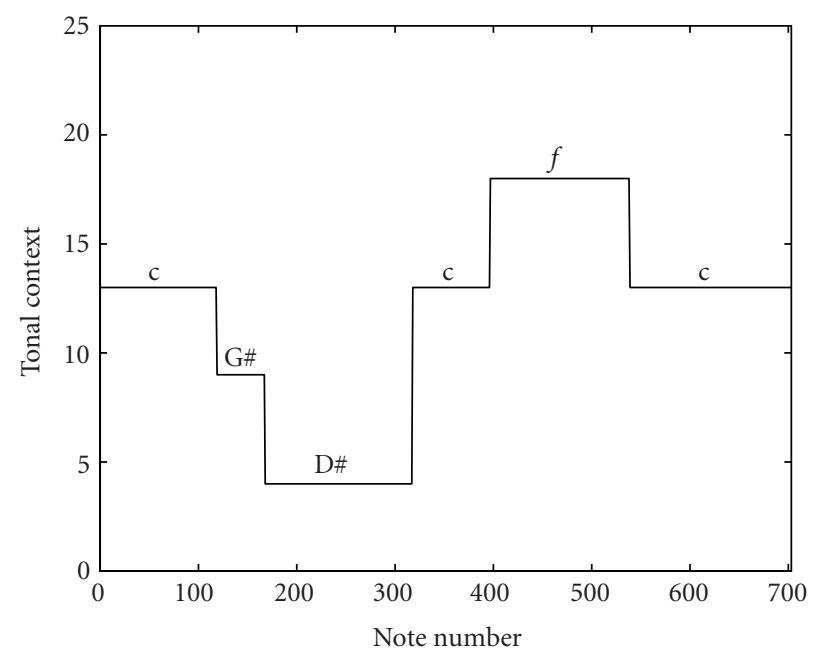

FIGURE 3: The result of applying the proposed estimation method to the sequence of key assignments for Bach's Prelude in C Minor from Book II of the Well-Tempered Clavier. Upper-case and lowercase letters represent major and minor keys, respectively. The length of the window is 99 .

introducing any biases toward small or large distances. Note that while no ordering of the keys was assumed, for purposes of visualization Figure 3 displays the output in the same way as Figure 2.

In the study by Krumhansl, two music theorists were independently asked to analyze the prelude by Bach on a measure-by-measure basis [14]. The experts indicated the primary key as well as keys of lesser strengths. The sequence of key assignments produced by the key-finding algorithm and the estimation method is almost identical to the judgements made by both experts. In cases where our algorithm disagrees with the choice of primary key made by the experts, 
it usually agrees with their indicated keys of lesser strength. Thus, we can conclude that this approach to localized key finding produces sensible assignments of keys. It also has the advantage that it allows the determination of the key in an arbitrary position in the score, that is, the algorithm does not depend on any subdivisions such as measures. ${ }^{1}$

\section{CASE STUDY 2: CORRECTION OF MISCLASSIFICATIONS IN PRIMARY LOCAL RECOGNITION OF IMAGE FEATURES}

In this case study, the proposed estimation method is applied in 2D for correction of misclassifications following classification (primary local recognition) of simulated remotely sensed data. First, in Section 4.1, classification of remotely sensed data is discussed and a method for performing this task is introduced. Then, the requirements for correction of misclassifications are discussed in Section 4.2. In contrast to the case study of Section 3, in this case there appears to be no theory from which the proximity matrix employed by the estimation method could be deduced directly, and therefore, in Section 4.3 we employ training-based optimization with genetic algorithms as the learning rule for finding the optimal proximity matrix. The accuracy of the obtained classification results is assessed in Section 4.4.

\subsection{Classification of remotely sensed data}

Remote-sensing systems are widely used for various applications providing valuable data for ecology, hydrology, agriculture, and other fields $[13,24,25]$. These systems commonly include several subsystems that operate in different bands of electromagnetic waves (optical, infrared, microwave). Since the obtained images can cover earth terrains with areas of thousands of square kilometers and the linear resolution of the imaging system is typically of the order of a few meters, the obtained images usually contain a very large number (millions) of pixels. Moreover, during many space missions the obtained remotely sensed data have been stored in huge databases, and since some of these missions still continue and other missions are planned in the near future, it is expected that the amount of available data will continue to grow rapidly.

However, the process of obtaining remotely sensed data is only an intermediate step in the course of extracting useful information from these data. In this sense, substantial challenges arise in processing the obtained multispectral or multichannel images $[24,25]$ and ensuring reliable, accurate, and appropriately fast extraction of valuable information $[26,27]$. It is not reasonable to expect that the required information can be extracted by human experts using visual analysis of the obtained images even if they have at their disposal some advanced image processing tools.

One way of addressing this problem is to apply automatic procedures of image classification that can be multi-

\footnotetext{
${ }^{1}$ Some composers, such as Eric Satie, have chosen to abandon the use of measures altogether in some of their compositions.
}

stage or iterative [28]. However, in practice even the most advanced techniques of automatic classification are unable to provide image classification without errors (misclassifications) $[26,27]$. The main reason is the inherent presence of noise, especially as the characteristics of noise vary widely for different types of images and components of multichannel data $[29,30]$. The correction of misclassifications is thus crucial.

Several versions of possible classification procedures have been developed in $[31,32,33,34]$. In such procedures, the classification is performed locally and is thus referred to as primary local recognition (PLR). The goal is to classify the central pixel in the sliding window into one of six possible classes:

(i) homogeneous region $(\mathrm{H})$;

(ii) edge between two homogeneous regions having different intensity means $(\mathrm{E})$;

(iii) neighborhood of a spike, that is, there is a spike in the sliding window, but not in the central pixel (NS);

(iv) spike in the central pixel of the sliding window-this corresponds to impulsive noise, that is, outliers (S);

(v) small-sized or prolonged object in the central pixelthese are characterized by compactness and connectedness of pixels as well as by homogeneity of values $(\mathrm{O})$;

(vi) neighborhood of a small-sized object, that is, there is a small-sized or prolonged object in the sliding window, but the central pixel does not belong to it (NO).

This kind of PLR can be very useful for solving many important practical tasks. These include edge detection needed for image segmentation [29] and small-sized object detection required for joint registration of images [35] and automatic target recognition [36]. Another task is the detection and removal of outliers. Moreover, after edges and small-sized objects have been detected and localized, it becomes possible to improve image classification in another classification scheme by taking into account neighboring pixels. In other words, PLR results can be useful in further classification of the same scene into, for example, land cover categories such as "forest," "water," and "urban" [30]. Specifically, after detection of edges and, possibly, their thinning, it is intuitively clear that one is in a better position to determine the subset of pixels that correspond to two homogeneous regions forming the considered edge. Similarly, after detection of small-sized objects, the corresponding pixels can be united, allowing for further classification of the considered small-sized objects by applying recognition algorithms that take into account the spatial features of the small-sized object.

The window size $5 \times 5$ samples is used in the PLR algorithms. This size is large enough for reliable classification of the central pixel, and at the same time it is small enough to avoid ambiguity in the classification of complex situations. The PLR method is based on the calculation of six local statistical parameters for each position of the sliding window. The classifier, an expert system [34] or a neural network $[31,32,33]$, then makes the decision on the class 
membership of the central pixel in the window by using these parameters as features. The details can be found in the aforementioned papers. In this case study, we employ the approach described in [31].

Throughout this case study the image shown in Figure 4 is used as a test image. The image is called "Mosaic" and it is a synthetic 8 -bit image of size $256 \times 256$. In the image, there is a homogeneous region in the lower part, and in the middle horizontal part there are small-sized objects having different shapes and different numbers of pixels belonging to them. Their contrasts with respect to the background vary as well. In the upper part there are homogeneous regions inside and between many mosaic-type objects with different shapes, orientations, and contrasts. The idea behind this image is to simulate such situations that typically occur in remotely sensed images.

The primary local recognition stage as well as the used noise model are discussed in detail in [31]. Here it suffices to say that multiplicative Gaussian noise with unit mean and variance $\sigma^{2}=0.003$ was added to the Mosaic image before PLR. ${ }^{2}$ The result after PLR is shown in Figure 5. Although there are no spikes in the Mosaic image of Figure 4, there are a lot of pixels for which the PLR algorithm assigns the class NS. This happens due to the fact that classification is done locally. For example, when the sliding window is approaching a small-sized object, the sliding window may contain only one pixel belonging to that object. If all the other 24 pixels have approximately the same intensity level, the central pixel is classified into NS. Similarly, when the sliding window contains more than one pixel belonging to a small-sized object but the central pixel does not belong to the object, the classification is appropriately NO. These situations occur also with edges (E) between homogeneous regions $(\mathrm{H})$, and thus edges, too, are surrounded by NS as well as NO pixels.

\subsection{Requirements for correction of misclassifications}

The following requirements are defined for the correction of misclassifications after primary classification:

(R1) E and O pixels should be preserved if they are spatially grouped and they are surrounded by NS and NO pixels;

(R2) isolated pixels of $\mathrm{H}, \mathrm{E}$, and $\mathrm{O}$ should be removed;

(R3) all pixels with initial classification NS, S, or NO should be removed.

Since all pixels with initial classification NS, S, or NO should be removed, these classes are considered as supplementary classes, that is, belonging to the multiset $B_{s}$ (see Section 2). In fact, their primary purpose is to assist in misclassification correction, and as can be seen by looking at requirements (R1) and (R2), the distinction between correctly recognized and misclassified $\mathrm{E}$ and $\mathrm{O}$ pixels is made primarily on the basis of whether they are surrounded by supplementary classes

\footnotetext{
${ }^{2}$ It is shown in [5] that the proposed estimation method can be applied successfully with higher noise intensities as well.
}

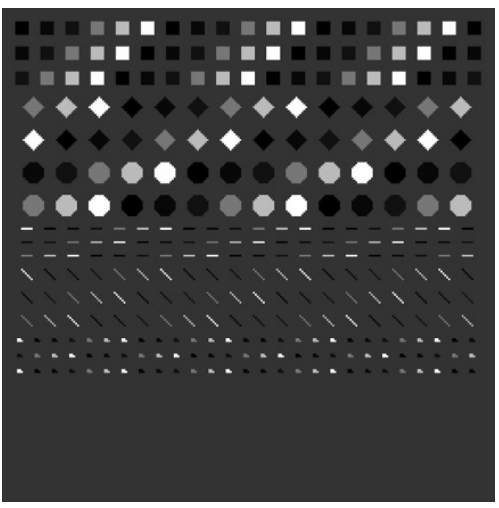

Figure 4: The Mosaic test image.

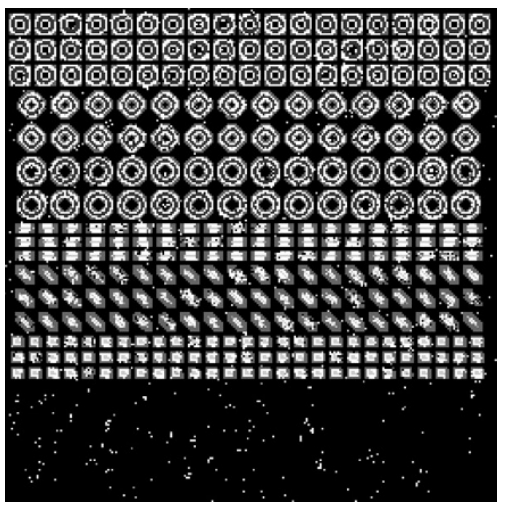

FIgure 5: The PLR result of the noisy Mosaic image. The classes $\mathrm{H}$, E, NS, S, O, and NO are shown by gradations of gray from black to white.

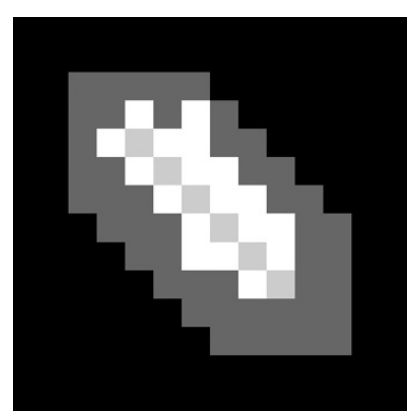

Figure 6: An example of how correctly classified O pixels are surrounded by NS and NO pixels. The classes are $\mathrm{H}, \mathrm{NS}, \mathrm{O}$, and NO, and are shown by gradations of gray from black to white.

or not. Specifically, correctly classified $\mathrm{E}$ and $\mathrm{O}$ pixels are surrounded by both NS pixels (more distantly) and NO pixels (more closely), whereas this is unlikely to occur for misclassified $\mathrm{E}$ and $\mathrm{O}$ pixels. This property is illustrated in Figure 6. The classes $\mathrm{H}, \mathrm{E}$, and $\mathrm{O}$ are considered as basic classes, that is, belonging to the multiset $B_{b}$.

Before we are ready to apply the proposed estimation method to the PLR data of Section 4.1, we need to fix 
a number of parameters. The above division of classes into basic and supplementary ones leads to the use of (10) (with $p=1)$ as the processing operator. As explained above, here $B_{b}=\{\mathrm{H}, \mathrm{E}, \mathrm{O}\}$ and $B_{s}=\{\mathrm{NS}, \mathrm{S}, \mathrm{NO}\}$. Further, since a $5 \times 5$ window is used in the PLR stage, it is natural to use a $5 \times 5$ window here as well. This is because, ideally, a $5 \times 5$ neighborhood of an $\mathrm{E}$ or $\mathrm{O}$ pixel contains those supplementary class (NS and NO) pixels that are meant to assist in correction of misclassifications. A larger window would include many $\mathrm{H}$ pixels, and correction of misclassifications would be more difficult. A smaller window, in turn, would not include all the information contained in the supplementary classes.

For simplicity, only the central sample is weighted with the spatial weight $a=10$. The weighting of the central sample increases the probability of the central sample remaining as the output. This is desired, since it decreases the probability of a pixel that has been correctly classified in the PLR stage to be misclassified. However, at the same time the probability that a misclassified pixel remains misclassified increases, and therefore, center weighting is a compromise between detail preservation and misclassification correction.

\subsection{Training-based optimization}

The performance of the proposed method relies on a successful choice of the proximity matrix. In this case study, our approach for determining the proximity matrix is to adopt the learning or, as it is known in signal processing, the trainingbased optimization paradigm. In this framework, it is implicitly assumed that we can inductively learn from experience and make useful decisions for cases that we have not experienced before. Thus, we must employ an empiricist epistemology, but be able to do so in the presence of uncertainty. This entails making the assumption that different signals and images, despite exhibiting a range of characteristics, will share certain very general properties from which we can make inductive inferences. In this case study, all images contain different homogeneous regions, small-sized objects, and so on. These characterizations, although very general and unrestrictive, already inherently impose certain structural regularity that can be exploited in the process of learning. For instance, an image containing homogeneous regions $(\mathrm{H})$ and smallsized objects $(\mathrm{O})$ intrinsically implies that a pixel believed to belong to a small-sized object and yet surrounded entirely by pixels belonging to a homogeneous region is likely to be misclassified.

In general, the size of the search space for the optimization algorithm, that is, the number of possible proximity matrices, is too large for conventional calculus-based or enumerative optimization algorithms to be useful. In particular, in this case study, there are 18 elements in the proximity matrix and each can take 8 different values (see below), and thus there are $2^{21}$ candidates for the optimal proximity matrix. Moreover, there are typically various local optima in the search space and we therefore have to use a randomized optimization algorithm such as simulated annealing or a genetic algorithm (GA). Here, we choose to employ GAs, which are efficient search algorithms based on simulated evolution strategies [2].
Training-based optimization leads to a global combinatorial optimization problem, in which we have a pair $(S, f)$, where $S$ is a finite or countably infinite set of configurations and $f: S \mapsto \mathbb{R}$ is an optimization criterion. The set $S$ is also called the search space or the solution space. In the context of GAs, $f$ is to be maximized and is called the fitness function. The problem is to find a configuration $s_{0} \in S$ such that

$$
f\left(s_{0}\right) \geq f(s) \quad \forall s \in S .
$$

Thus,

$$
s_{0}=\arg \max _{s \in S} f(s) .
$$

Naturally, minimization problems can be considered similarly. The fitness function depends on the particular application [37].

The goal in the training phase is to find a proximity matrix that can be used in correction of misclassifications for a wide range of classified images. The training-based approach also provides a well-defined design methodology. We use a GA that is taken from [2] as the optimization algorithm. We use a fixed population size of 30 individuals. Since the population size is fixed, two offspring are produced in the recombination step. The mutation rate is such that each feature of the offspring can mutate independently with probability 0.03 .

The choice of a representative training pair (source image and target image) is crucial. Naturally, since the ultimate goal is to process real remotely sensed images, it would be good to be able to train the method on some representative real radar images. However, for such images it can be difficult to accurately determine the ground truth that is needed for the target image [26]. Therefore, we use the Mosaic image, which is a synthetic image for which the correct classification result can be readily obtained in the noise-free case by using an algorithm that is based on simple decision rules. For details of this algorithm, see [6]. The target image is shown in Figure 7 and the source image is shown in Figure 5. The properties of the Mosaic image have already been discussed in Section 4.1. Here, it suffices to say that the image represents a wide range of situations that can occur in practice in correction of misclassifications and is therefore a suitable training image.

Obviously, the choice of the fitness function is important as well. In our case, the set of all configurations $S$ is the set of all possible proximity matrices, and the only input parameter of the fitness function is a candidate $w^{\prime} \in S$ for the optimal proximity matrix (the initial candidate is chosen randomly). The real number to be maximized is then $f\left(w^{\prime}\right)$. First, the estimation method is applied to the source image using the candidate proximity matrix $w^{\prime}$. Then, $f\left(w^{\prime}\right)$ is the number of pixels in the source image that agree with the corresponding pixels in the target image.

We choose to use 3 bits to represent each element of the proximity matrix, which means that each of them can take 8 different values. The bounds can be chosen arbitrarily, and since natural numbers are easier to manipulate than real numbers, we choose $w_{i j} \in[0,7]$ for all pairs of $i$ and $j$. With 


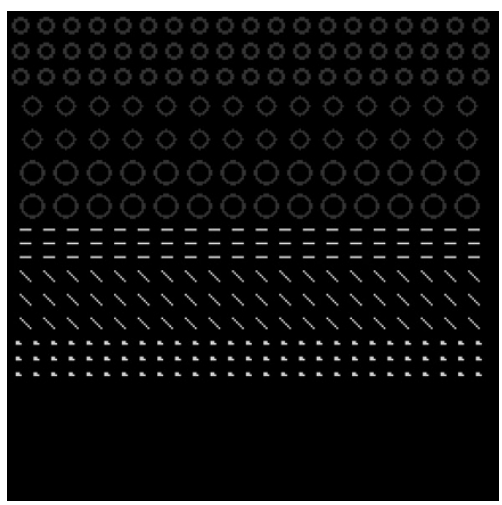

FIgURE 7: The Mosaic target image for training-based optimization. The classes are $\mathrm{H}$ (black), E (gray), and O (white).

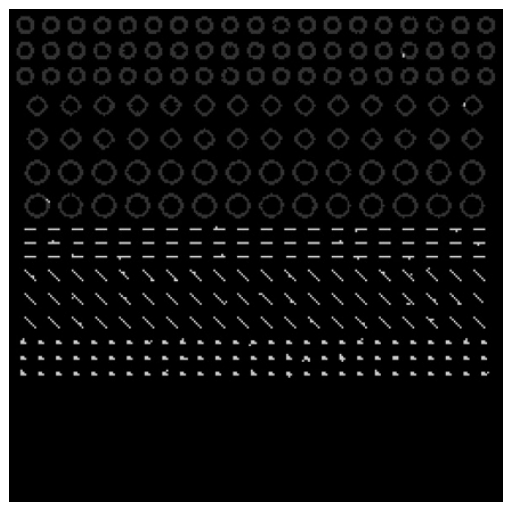

FIgURE 8: The Mosaic image after the application of the proposed estimation method. The classes are $\mathrm{H}$ (black), E (gray), and $\mathrm{O}$ (white).

these choices the solution space for the GA is small enough in order for the GA to converge and at the same time it is large enough for the GA to find a satisfactory optimization result. The training phase results in the optimal proximity matrix

$$
\mathbf{W}_{\text {opt }}=\left[\begin{array}{cccccc}
\text { H } & \text { E } & \text { NS } & \text { S } & \text { O } & \text { NO } \\
0 & 4 & 6 & 7 & 6 & 1 \\
7 & 0 & 6 & 5 & 5 & 3 \\
- & - & - & - & - & - \\
- & - & - & - & - & - \\
6 & 7 & 7 & 1 & 1 & 3 \\
- & - & - & - & - & -
\end{array}\right] .
$$

Because a pixel representing one of the supplementary classes S, NS or, NO cannot be an output of (10), the proximities in the third, fourth, and sixth rows do not have an effect on the output of the estimation method, and therefore do not undergo optimization. To emphasize this, those proximities are labeled as "-" in the matrix above.

\subsection{Simulations and assessment of accuracy}

In the PLR result of the Mosaic image there are 1070 misclassified pixels to be corrected (see Figure 5). Further, there are 19494 pixels representing supplementary classes, which need to be removed in the misclassification correction stage. When the estimation method is applied to the PLR result using the matrix (16), the image shown in Figure 8 is obtained. As can be seen, many of the misclassifications have been corrected. To be exact, 733 misclassifications remain, and all the supplementary classes have been removed leading to the overall accuracy of 0.989 , that is, 98.9 percent of all pixels are classified correctly. Most of the misclassifications are related to edge detection, that is, 668 misclassifications result from an E pixel being committed to the $\mathrm{H}$ class or vice versa. However, as there are only 2 discontinuities in edges, this is not a serious problem. The remaining 65 misclassifications are related to recognition of small-sized objects $(\mathrm{O})$.

The number of corrected misclassifications in the Mosaic image is 498 , that is, 46.5 percent of all misclassifications are corrected. It is also worth mentioning that all misclassifications, even those that are spatially grouped, are removed from the bottom part of the image in which there is a large homogeneous region. Further, only 6 pixels that are initially recognized correctly in the PLR stage are misclassified by the estimation method. The number of pixels representing supplementary classes that are replaced by a wrong class is also relatively low, 155 .

An example of edge detection and correction of misclassifications in an edge neighborhood is illustrated in Figure 9. A portion of the Mosaic image is presented in Figure 9a. Correspondingly, Figure $9 \mathrm{~b}$ shows the target image for classification, Figure 9c shows the PLR result, and Figure 9d shows the result after the application of the proposed estimation method. We see that although the edge is recognized reasonably well in the PLR stage, in addition to many pixels representing supplementary classes, there are three small-sized object pixels that are clearly misclassifications. Two of these are located in the top right corner of the edge and one in the bottom right corner. Two of these three misclassifications are corrected by estimation method, and at the same time the edge remains continuous. Similarly, an example of smallsized object detection and correction of misclassifications in a small-sized object neighborhood is presented in Figure 10. In this case, the PLR stage results in an image in which two pixels belonging to the small-sized object are misclassified as spike pixels. The result after the application of the estimation method, however, is a perfect classification as can be seen by noting that Figure 10d is identical to Figure 10b.

It is natural that a good misclassification correction result is obtained when the estimation method is applied to the image on which it was trained. The real test is to analyze its performance when other images are used. First, we need to define a concept called the error matrix. The error matrix is a square array of numbers that express the number of pixels assigned to a particular category in a classification relative to the number of pixels assigned to a particular category in the reference data. Here, we use the correct classification result as 


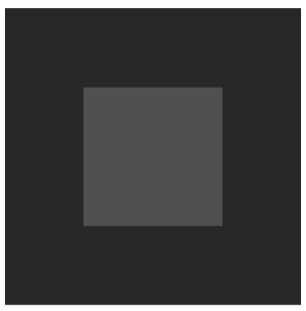

(a)

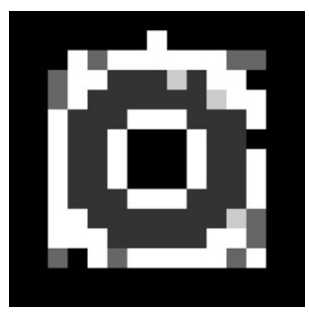

(c)

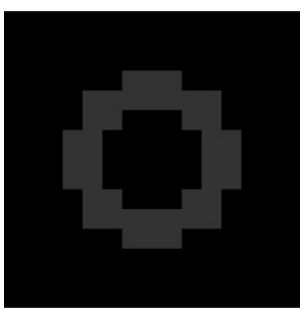

(b)

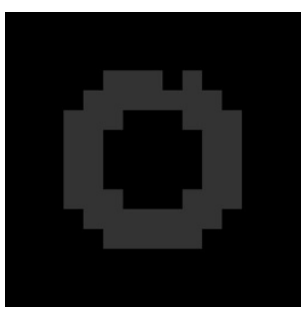

(d)
FIGURE 9: An example of correction of misclassifications in the neighborhood of an edge. (a) A portion of the original Mosaic image, (b) the target image for the portion, (c) the portion after the PLR stage, (d) the portion after the application of the proposed estimation method. In (b), (c), and (d) the classes H, E, NS, O, and $\mathrm{NO}$ are shown by gradations of gray from black to white.

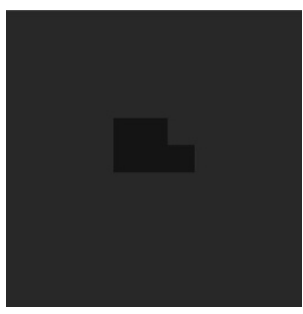

(a)

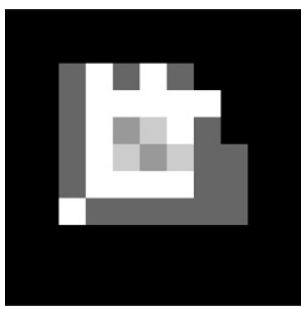

(c)

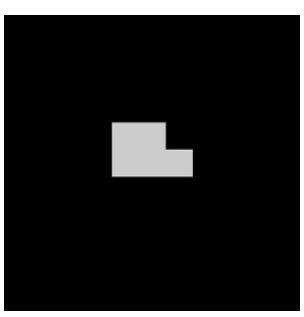

(b)

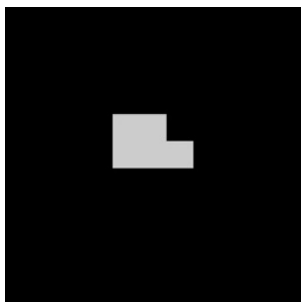

(d)
FIGURE 10: An example of correction of misclassifications in the neighborhood of a small-sized object. (a) A portion of the original Mosaic image, (b) the target image for the portion, (c) the portion after the PLR stage, (d) the portion after the application of the proposed estimation method. In (b), (c), and (d) the classes H, NS, S, $\mathrm{O}$, and $\mathrm{NO}$ are shown by gradations of gray from black to white.

the reference data. The columns represent the reference data whereas the rows indicate the classification whose accuracy is to be assessed. The error matrix can be given as

$$
\mathbf{E}=\left[\begin{array}{cccc|c}
n_{11} & n_{12} & \cdots & n_{1 k} & n_{1+} \\
n_{21} & n_{22} & \cdots & n_{2 k} & n_{2+} \\
\vdots & \ddots & \ddots & \vdots & \vdots \\
n_{k 1} & n_{k 2} & \cdots & n_{k k} & n_{k+} \\
\hline n_{+1} & n_{+2} & \cdots & n_{+k} & n
\end{array}\right]
$$

where $k$ is the number of classes used by the classifier, $n_{i j}$ give the number of pixels that are classified into category $i$ when the correct classification is category $j, n_{i+}$ are the row sums, $n_{+j}$ are the column sums, and $n$ is the sum of all entries $n_{i j}$. The numbers of correctly classified pixels are given on the main diagonal, that is, when $i=j[26]$.

An assessment of classification accuracy can be done using a statistical method called Kappa analysis [26]. It is a discrete multivariate technique that is used in accuracy assessment for statistically determining if an error matrix is significantly different from another error matrix [38]. The technique was first introduced by Cohen [39] in 1960, and has since then been used in, for example, sociology and psychology. It was first published in a remote-sensing journal in 1983 [40], and has since then found popularity in the remote-sensing community. The motivation behind using a statistical-assessment tool such as Kappa analysis is that the overall accuracy, that is, the number of correctly classified pixels divided by the total number of pixels, does not take into account the statistical aspect of the classification result. In particular, in calculating the overall accuracy, the fact that there can be only a small number of pixels representing some class whereas for some other class the number can be very high is not taken into account. In the extreme case, the total number of pixels representing some class can be low enough in comparison to the total number of pixels in the image under classification for a good overall accuracy to be obtained even if most pixels belonging to that class are misclassified. This does not mean, however, that it is not important to be able to obtain a good classification of pixels belonging to some class that has only a few representatives.

Kappa analysis puts an approximately equal importance on the correct classification of all classes regardless of the number of pixels representing each class. The result of performing a Kappa analysis is the maximum likelihood estimate of the Kappa statistic called KHAT and denoted by $\hat{K}$. The meaning of the Kappa statistic is that it is a measure of the difference between the actual agreement between the reference data and an automated classifier and the chance agreement between the reference data and a random classifier. In other words, the Kappa statistic tells us how statistically significant the improvement in classification accuracy provided by the classifier under assessment is when the classifier is compared against a random classifier. In practice, we can calculate the KHAT statistic using the formula

$$
\hat{K}=\frac{n \sum_{i=1}^{k} n_{i i}-\sum_{i=1}^{k} n_{i+} n_{+i}}{n^{2}-\sum_{i=1}^{k} n_{i+} n_{+i}} .
$$


The KHAT statistic is asymptotically normally distributed even though the data in the error matrix are discrete [26].

Here, the aim of Kappa analysis is to establish that the optimal operator can be used successfully also outside the training data set, that is, that the estimation method with the proximity matrix (16) provides a statistically significant improvement in classification accuracy with images that are significantly different from the Mosaic image. Our set of images includes a total of eight images. All of these images are 8 -bit images of size $256 \times 256$. Like the Mosaic image, they contain edges (E) and small-sized objects (O) of many different shapes, sizes and contrasts. In particular, they contain shapes and sizes that do not appear in the Mosaic image. Moreover, in three of the images there are also prolonged objects, which are lines that are 1 pixel in thickness, and belong to the same class $(\mathrm{O})$ as small-sized objects.

We first apply PLR to all of the eight test images and build the corresponding error matrices. The resulting combined error matrix, that is, the sum of the eight error matrices is

$$
\mathbf{E}_{1}=\left[\begin{array}{ccc|c}
379284 & 0 & 0 & 379284 \\
654 & 22656 & 274 & 23584 \\
4341 & 464 & 10289 & 15094 \\
\hline 384279 & 23120 & 10563 & 417962
\end{array}\right] .
$$

Then, we apply the proposed estimation method to all of the eight PLR results and obtain the combined error matrix

$$
\mathbf{E}_{2}=\left[\begin{array}{ccc|c}
471676 & 467 & 85 & 472228 \\
939 & 22910 & 274 & 24123 \\
1137 & 115 & 10429 & 11681 \\
\hline 473752 & 23492 & 10788 & 508032
\end{array}\right]
$$

In these error matrices the first row represents the class $\mathrm{H}$, the second row represents the class E, and the third row represents the class $\mathrm{O}$. The grand total of pixels is greater in the second error matrix than in the first one. This is explained by the fact that before the application of the estimation method the image contains 90070 supplementary class pixels that are not accounted for in the first error matrix. The estimation method replaces all the pixels that represent supplementary classes by pixels that represent basic classes, and the grand total increases accordingly.

Examining the error matrices (19) and (20), we immediately notice that for each of the three classes the proportion of correctly classified pixels is increased. Specifically, the overall accuracy is increased by the application of the estimation method from 0.986 to 0.994 . We can also look at the ability of the estimation method to correct misclassifications and to avoid introducing new misclassifications in the case of our test images. First of all, the number of corrected misclassifications is appropriately high, 3863 (67.4 percent of all misclassifications), which means 483 corrected misclassifications per image on the average. On the other hand, the number of misclassifications that are introduced by the estimation method is appropriately low, 91, which means less than 12 new misclassifications per image. The number of supplementary class pixels that are replaced by a wrong class is also quite low, 1056, which means 132 cases per image on the average. These numbers mean that the number of misclassifications that are present in the test images is reduced from 5733 to 3017 by applying the estimation method. Moreover, all of the values are comparable with (and in many cases better than) the corresponding values for the Mosaic image.

Although the number of correctly classified pixels increases for all of the three classes, some of the numbers representing misclassifications, that is, off-diagonal values, increase as well. This is mainly due to the fact that some pixels that represent a supplementary class are misclassified by the estimation method. In spite of these misclassifications, the overall accuracy is increased by the estimation method. However, as explained above, the overall accuracy is not a reliable measure of the accuracy of a classification. We therefore calculate the KHAT values for the two error matrices and perform a Kappa analysis. The KHAT values can be calculated using (18), and we obtain $\hat{K}_{1}=0.9151$ and $\hat{K}_{2}=$ 0.9545 for the error matrices of before and after the application of the estimation method, respectively. Thus, like the overall accuracy, the KHAT value is higher after the application of the estimation method than before its application. In fact, the difference is greater in the KHAT values than in the overall accuracies, which means that the improvement in the classification result that is provided by the estimation method is more significant than the overall accuracies suggest.

Finally, hypothesis tests based on confidence intervals around the KHAT value can be made using the approximate large sample variance, which can be computed using the delta method (see, e.g., [26]). The hypothesis test to determine if two independent KHAT values $\hat{K}_{1}$ and $\hat{K}_{2}$, and therefore two error matrices, are significantly different from each other is based on the test statistic

$$
Z=\frac{\left|\hat{K}_{1}-\hat{K}_{2}\right|}{\sqrt{\operatorname{var}\left(\hat{K}_{1}\right)+\operatorname{var}\left(\hat{K}_{2}\right)}},
$$

where $\operatorname{var}\left(\hat{K}_{i}\right)$ denotes the approximate large sample variance of $\hat{K}_{i}$. The statistic $Z$ is standardized and normally distributed. Given the null hypothesis $H_{0}:\left(K_{1}-K_{2}\right)=0$ and the alternative hypothesis $H_{1}:\left(K_{1}-K_{2}\right) \neq 0, H_{0}$ is rejected if $Z \geq Z_{\alpha / 2}$, where $\alpha / 2$ is the confidence level of the two-tailed $Z$ test.

With this test, it is possible to statistically compare the classification results before and after correction of misclassifications. We obtain $\operatorname{var}\left(\hat{K}_{1}\right)=1.2087 \cdot 10^{-6}$ and $\operatorname{var}\left(\hat{K}_{2}\right)=$ $6.7457 \cdot 10^{-7}$. Thus, (21) gives $Z=28.68$. At the 99 percent confidence level, the critical value of the test statistic is $Z_{\alpha / 2}=2.58$. Thus, the obtained value of $Z$ is greater than the critical value and the null hypothesis is rejected. Therefore, we conclude that the proposed estimation method provides a statistically significant improvement in classification accuracy. 


\section{CONCLUSIONS}

An estimation method for correction of misclassifications was presented. The method can be considered as a postclassification smoothing operator, because it is intended to be used to correct misclassifications that appear in the output of a classification algorithm based on local context information. It can be applied to any signal that is comprised of class data regardless of its dimensionality.

Two case studies were presented to illustrate the performance of the proposed method. These case studies are different in many ways. The most notable distinction is that the first takes its example from music and applies the method in $1 \mathrm{D}$, whereas the second applies it in 2D for correcting misclassifications in simulated remotely sensed images. Further, the approach to finding a suitable proximity matrix is different in the case studies. In the first, the proximity matrix is derived directly from music perception studies, whereas in the second, training-based optimization using genetic algorithms is employed. The applications of the case studies also require significantly different numbers of classes 24 and 6, respectively. The sizes of the sliding window are also significantly different being 99 and 25 samples, respectively. Nonetheless, the proposed method is found to provide a good misclassification correction result in both case studies.

\section{ACKNOWLEDGMENTS}

The support of the Academy of Finland is gratefully acknowledged. V. V. Lukin is supported by the Project Grant 1659 of the Scientific Technology Center of Ukraine. A. N. Dolia is supported by Engineering and Physical Science Research Council Grant GR/R61215, UK, and by Data and Information Fusion, Defence Technology Center Grant, UK.

\section{REFERENCES}

[1] R. Dubes and A. K. Jain, "Random field models in image analysis," J. Appl. Stat., vol. 16, no. 2, pp. 131-164, 1989.

[2] D. Goldberg, Genetic Algorithms in Search, Optimization, and Machine Learning, Addison-Wesley, Reading, Mass, USA, 1989.

[3] A. H. S. Solberg, T. Taxt, and A. K. Jain, "A Markov random field model for classification of multisource satellite imagery," IEEE Transactions on Geoscience and Remote Sensing, vol. 34, no. 1, pp. 100-113, 1996.

[4] V. V. Lukin, I. Shmulevich, O. P. Yli-Harja, and A. N. Dolia, "Correction of misclassifications in primary local image recognition using a nonlinear graph-based estimation technique," in Image and Signal Processing for Remote Sensing VI, S. Serpico, Ed., vol. 4170 of Proceedings of SPIE, pp. 251-259, Barcelona, Spain, January 2001.

[5] A. Niemistö, V. V. Lukin, A. N. Dolia, O. P. Yli-Harja, and I. Shmulevich, "Misclassification correction in primary local recognition of component images of multichannel remote sensing data," in Image and Signal Processing for Remote Sensing VII, S. Serpico, Ed., vol. 4541 of Proceedings of SPIE, pp. 134-145, Toulouse, France, September 2001.
[6] A. Niemistö, V. V. Lukin, I. Shmulevich, O. P. Yli-Harja, and A. N. Dolia, "A training-based optimization framework for misclassification correction," in Proc. 12th Scandinavian Conference on Image Analysis (SCIA '01), pp. 691-698, Bergen, Norway, June 2001.

[7] O. P. Yli-Harja and I. Shmulevich, "Correcting misclassifications in hyperspectral image data using a nonlinear graphbased estimation technique," in Proc. International Symposium on Nonlinear Theory and Its Applications (NOLTA '99), pp. 259-262, Waikoloa, Hawaii, USA, November 1999.

[8] O. P. Yli-Harja, I. Shmulevich, and K. Lemström, "Graphbased smoothing of class data with applications in musical key finding," in Proc. IEEE-EURASIP Workshop on Nonlinear Signal and Image Processing, pp. 311-315, Antalya, Turkey, June 1999.

[9] D. Johnson and W. Wang, "Symbolic signal processing," in Proc. IEEE Int. Conf. Acoustics, Speech, Signal Processing (ICASSP '99), pp. 1361-1364, Phoenix, Ariz, USA, March 1999.

[10] F. R. McMorris, H. M. Mulder, and R. C. Powers, "The median function on median graphs and semilattices," Discrete Applied Mathematics, vol. 101, no. 1-3, pp. 221-230, 2000.

[11] F. R. McMorris and R. C. Powers, "The median procedure in a formal theory of consensus," SIAM J. Discrete Math., vol. 8, no. 4, pp. 507-516, 1995.

[12] J. T. Astola and P. Kuosmanen, Fundamentals of Nonlinear Digital Filtering, CRC Press, Boca Raton, Fla, USA, 1997.

[13] T. Lillesand and R. Kiefer, Remote Sensing and Image Interpretation, John Wiley \& Sons, New York, NY, USA, 1994.

[14] C. Krumhansl, Cognitive Foundations of Musical Pitch, Oxford University Press, New York, NY, USA, 1990.

[15] S. Holtzman, "A program for key determination," Interface, vol. 6, no. 1, pp. 29-56, 1977.

[16] H. Longuet-Higgins and M. Steedman, "On interpreting Bach," in Machine Intelligence, B. Meltzer and D. Michie, Eds., vol. 6, pp. 221-241, University of Edinburgh Press, Edinburgh, Scotland, 1971.

[17] P. Vos and E. van Geenen, "A parallel-processing key-finding model," Music Perception, vol. 14, no. 2, pp. 185-224, 1996.

[18] E. J. Coyle and I. Shmulevich, "A system for machine recognition of music patterns," in Proc. IEEE Int. Conf. Acoustics, Speech, Signal Processing (ICASSP '98), pp. 3597-3600, Seattle, Wash, USA, May 1998.

[19] I. Shmulevich and O. P. Yli-Harja, "Localized key-finding: Algorithms and applications," Music Perception, vol. 17, no. 4, pp. 531-544, 2000.

[20] I. Shmulevich, O. Yli-Harja, E. Coyle, D.-J. Povel, and K. Lemström, "Perceptual issues in music pattern recognition: Complexity of rhythm and key finding," Computers and the $\mathrm{Hu}$ manities, vol. 35, no. 1, pp. 23-35, 2001.

[21] I. Shmulevich and E. J. Coyle, "The use of recursive median filters for establishing the tonal context in music," in Proc. IEEE Workshop on Nonlinear Signal and Image Processing, Mackinac Island, Mich, USA, 1997.

[22] C. Krumhansl and E. Kessler, "Tracing the dynamic changes in perceived tonal organization in a spatial representation of musical keys," Psychological Review, vol. 89, no. 4, pp. 334368, 1982.

[23] J. Kruskal and M. Wish, Multidimensional Scaling, Sage Publications, Beverly Hills, Calif, USA, 1978.

[24] W. Keydel, "SAR technique and technology, its present state of the art with respect to user requirements," in Proc. 1st European Conf. Synthetic Aperture Radar, pp. 19-24, Königswinter, Germany, March 1996.

[25] E. Stofan, D. Evans, C. Schmullius, et al., "Overview of results of Spaceborne Imaging Radar-C, X-Band Synthetic Aperture 
Radar (SIR-C/X-SAR)," IEEE Transactions on Geoscience and Remote Sensing, vol. 33, no. 4, pp. 817-828, 1995.

[26] R. Congalton and K. Green, Assessing the Accuracy of Remotely Sensed Data: Principles and Practices, CRC Press, Boca Raton, Fla, USA, 1999.

[27] D. Landgrebe, "Information extraction principles and methods for multispectral and hyperspectral image data," in Information Processing for Remote Sensing, C. H. Chen, Ed., pp. 3-38, World Scientific Publishing, River Edge, NJ, USA, 1999.

[28] B. Solaiman, L. Pierce, and F. Ulaby, "Multisensor data fusion using fuzzy concepts: application to land-cover classification using ERS-1/JERS-1 SAR composites," IEEE Transactions on Geoscience and Remote Sensing, vol. 37, no. 3, pp. 1316-1326, 1999.

[29] J. Richards and X. Jia, Remote Sensing Digital Image Analysis: An Introduction, Springer-Verlag, Berlin, Germany, 1999, edited by W. Gessner and D. Ricken.

[30] V. V. Lukin, J. T. Astola, V. Melnik, et al., "Data fusion and processing for airborne multichannel system of radar remote sensing: Methodology, stages, and algorithms," in Sensor Fusion: Architectures, Algorithms, and Applications IV, B. Dasarathy, Ed., vol. 4051 of Proceedings of SPIE, pp. 215226, Orlando, Fla, USA, April 2000.

[31] A. N. Dolia, A. Burian, V. V. Lukin, C. Rusu, A. A. Kurekin, and A. A. Zelensky, "Neural network application for primary local recognition and nonlinear adaptive filtering of images," in Proc. 6th IEEE Conference on Electronics, Circuits and Systems, pp. 847-850, Pafos, Cyprus, September 1999.

[32] A. N. Dolia, V. V. Lukin, A. Kurekin, A. Zelensky, N. Ponomarenko, and A. Sokolov, "Modified image local recognition system for locally adaptive filtering," in Proc. 6th Int. Symp. Automatic Control and Computer Science, pp. 84-89, Iasi, Romania, November 1998.

[33] A. N. Dolia, V. V. Lukin, A. Zelensky, J. T. Astola, and C. Anagnostopoulos, "Neural networks for local recognition of images with mixed noise," in Applications of Artificial Neural Networks in Image Processing VI, N. Nasrabadi and A. Katsaggelos, Eds., vol. 4305 of Proceedings of SPIE, pp. 108-118, San Jose, Calif, USA, January 2001.

[34] V. V. Lukin, N. Ponomarenko, J. T. Astola, and K. Saarinen, "Algorithms of image nonlinear adaptive filtering using fragment recognition by expert system," in Nonlinear Image Processing VII, E. R. Dougherty, J. T. Astola, and H. G. Longbotham, Eds., vol. 2662 of Proceedings of SPIE, pp. 179-190, San Jose, Calif, USA, March 1996.

[35] G. P. Kulemin, V. V. Lukin, A. A. Zelensky, A. A. Kurekin, and E. T. Engman, "Soil-erosion-state interpretation using pre- and postprocessing of multichannel radar images," in Remote Sensing for Agriculture, Ecosystems, and Hydrology II, E. T. Engman, Ed., vol. 3499 of Proceeding of SPIE, pp. 134144, Barcelona, Spain, December 1998.

[36] F. Sadjadi, H. Nasr, H. Amehdi, and M. Bazakos, "Knowledgeand model-based automatic target recognition algorithm adaptation," Optical Engineering, vol. 30, no. 2, pp. 183-188, 1991.

[37] P. Koivisto, H. Huttunen, and P. Kuosmanen, "Training-based optimization of soft morphological filters," Journal of Electronic Imaging, vol. 5, no. 3, pp. 300-322, 1996.

[38] Y. Bishop, S. Fienberg, and P. Holland, Discrete Multivariate Analysis: Theory and Practice, MIT Press, Cambridge, Mass, USA, 1980.

[39] J. Cohen, "A coefficient of agreement for nominal scales," Educ. Psychol. Meas., vol. 20, no. 1, pp. 37-46, 1960.

[40] R. G. Congalton and R. A. Mead, "A quantitative method to test for consistency and correctness in photo-interpretation,"
Photogrammetric Engineering and Remote Sensing, vol. 49, no. 1, pp. 69-74, 1983.

Antti Niemistö was born in Tampere, Finland in 1977. He received the M.S. degree with honours in computer science in 2002 from Tampere University of Technology, Tampere, Finland. From 2002 to present he has been a Ph.D. student at the same university. In 2003 and 2004 he was a Research Intern at The University of Texas M. D. Anderson Cancer Center, Houston, Texas, USA. Currently he is a Researcher with the Institute of Signal Processing of Tampere University of Technology. His research interests include nonlinear signal and image processing, image analysis, and bioinformatics.

Ilya Shmulevich received the Ph.D. degree in electrical and computer engineering from Purdue University, West Lafayette, Indiana in 1997. From 1997 to 1998, he was a postdoctoral researcher at the $\mathrm{Ni}$ jmegen Institute for Cognition and Information, University of Nijmegen, Nijmegen, the Netherlands, and National Research Institute for Mathematics and Computer Science, University of Amsterdam, Amster-

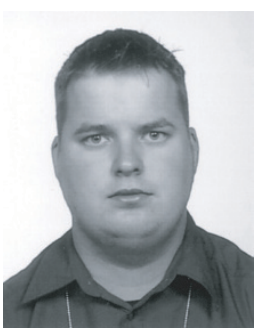
dam, the Netherlands, where he studied computational models of music perception and recognition. From 1998 to 2000, he was a senior researcher at the Tampere International Center for Signal Processing, Signal Processing Laboratory, Tampere University of Technology, Tampere, Finland. Presently, he is an Assistant Professor with the Cancer Genomics Laboratory, The University of Texas M. D. Anderson Cancer Center, Houston. His research interests include computational genomics, nonlinear signal and image processing, computational learning theory, and music recognition and perception.

Vladimir V. Lukin was born in 1960 in Belarus (former Soviet Union). He graduated in 1983 from the Faculty of Radioelectronic Systems, Kharkov Aviation Institute (now National Aerospace University), Kharkov, Ukraine, and received the Diploma degree with honours in radioengineering. Since then, he has been with the Department of Transmitters, Receivers, and Signal Processing of the same faculty. He received Can-

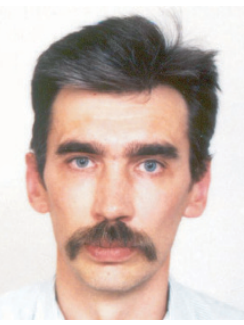
didate of Technical Science Diploma in radioengineering in 1988 and Senior Researcher Diploma in 1991. In 2003 he got the degree of Doctor of Technical Science in remote aerospace investigations. In the academic year 1992/1993 he was for 5 months a Visiting Researcher at Northern Jiaotong University, Beijing, China. Since 1995, he has been in cooperation with Tampere University of Technology (Institute of Signal Processing and TICSP). Since 1996, he has been in the Program Committee of Nonlinear Image Processing Conference, SPIE Symposium "Photonics West" in San Jose, USA. Since 1989, he has been Vice Chairman of the Department of Transmitters, Receivers, and Signal Processing. He has published more than 200 journal and conference papers, more than 90 of which are in English. 
Alexander N. Dolia received the M.S. degree with distinction in computer science from the Kharkov Aviation Institute (now National Aerospace University), Kharkov, Ukraine in 1994 and the Ph.D. degree in radio engineering from the National Aerospace University, Kharkov, Ukraine in 2001. From 1988 to 1989 he was an operator of optical tracking systems with the tracking and monitoring stations, USSR Air Forces,

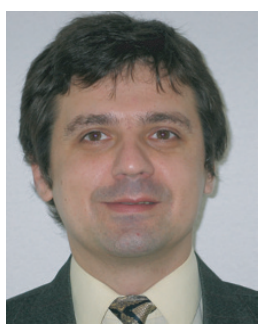
Priozersk, Kazakhstan. Since 1994 he has been associated with the Department of Transmitters, Receivers, and Signal Processing of the Faculty of Radio-Engineering Systems, National Aerospace University, Kharkov, Ukraine as a research fellow. From 2002 to 2003, he was a postdoctoral research fellow with the Department of Psychology, University of Glasgow, Scotland, where he studied computational models of visual cortex and human motion with emotions. Currently, he is a postdoctoral research fellow with the Electronics and Computer Science Department, University of Southampton, England. His research interests include target detection/classification and tracking, statistical machine learning, multisensor active management, data mining, bioinformatics, and vision.

Olli Yli-Harja received the Doctor of Technology degree in computer science and applied mathematics from Lappeenranta University of Technology, Finland, in 1989. During the years 1988-1998 he was a Research Scientist and a Lecturer in computer science, at the Technical Research Centre of Finland, Helsinki University of Technology, and University of Helsinki. In 1998 he was a research fellow at East Anglia University,

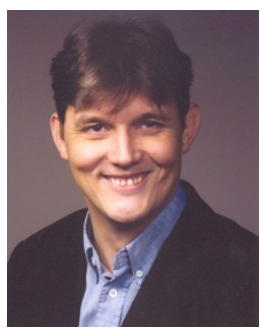
Norwich, UK. In 1998-2001 he was a Senior researcher at the Institute of Signal Processing in Tampere University of Technology. Currently, he is a Professor at the Institute of Signal Processing in Tampere University of Technology. His research interests include computational systems biology, music and perception, image analysis, complexity, and nonlinear filters. 